\title{
Correction to: Causes of recurrence in laparoscopic inguinal hernia repair
}

\author{
Manjunath Siddaiah-Subramanya ${ }^{1,2,8} \cdot$ Darius Ashrafi $^{2,3,4} \cdot$ Breda Memon $^{3,4} \cdot$ Muhammed Ashraf Memon $^{2,3,5,6,7,9}$
}

Published online: 27 September 2018

๑) Springer-Verlag France SAS, part of Springer Nature 2018

\section{Correction to: Hernia}

https://doi.org/10.1007/s10029-018-1817-x

In the original publication, affiliation 3 was incorrectly published for the author 'Darius Ashrafi'. The correct affiliation should read as 'Department of Surgery, Sunshine Coast University Hospital, Birtinya, QLD, Australia.

The original article can be found online at https://doi.org/10.1007/ s10029-018-1817-x.

Muhammed Ashraf Memon

mmemon@yahoo.com

Manjunath Siddaiah-Subramanya

manjunathbss9@yahoo.com

Darius Ashrafi

d.r.ashrafi@gmail.com

Breda Memon

bmemon@yahoo.com

1 Sir Charles Gairdner Hospital, Perth, Australia

2 Mayne Medical School, University of Queensland, Brisbane, QLD, Australia

3 Department of Surgery, Sunshine Coast University Hospital, Birtinya, QLD, Australia

4 South East Queensland Surgery (SEQS) and Sunnybank Obesity Centre, Sunnybank, QLD, Australia
5 Faculty of Health Sciences and Medicine, Bond University, Gold Coast, QLD, Australia

6 School of Agricultural, Computing and Environmental Sciences, International Centre for Applied Climate Science, University of Southern Queensland, Toowoomba, QLD, Australia

7 Faculty of Health and Social Science, Bolton University, Bolton, Lancashire, UK

8 School of Medicine, Griffith University, Nathan, QLD, Australia

9 South East Queensland Surgery and Sunnybank Obesity Centre, McCullough Centre, Suite 9, 259 McCullough Street, Sunnybank, QLD 4109, Australia 